\title{
非对称杂化的手性膦-亚磷酰胺酯配体在不对称催化反应中的应用进展
}

\author{
侯传金 $*, a, b$ 刘小宁 ${ }^{a}$ 夏 英 ${ }^{a}$ 胡向平 $*, b$ \\ $\left({ }^{a}\right.$ 大连工业大学轻工与化学工程学院 大连 116034) \\ ${ }^{b}$ 中国科学院大连化学物理研究所 大连 116023)
}

\begin{abstract}
摘要 非对称杂化的手性膦一亚磷酰胺酯配体因其合成简便、易于调控、结构稳定等优点, 被广泛应用于不对称催化反 应中, 如: 不对称氢化、不对称氢甲酰化、不对称烯丙基烷基化、不对称氢膦酰化、不对称 [3+2]-环加成、不对称 1,4加成和 1,4-还原反应等. 综述了手性膦-亚磷酰胺酯配体的种类、合成及其在不对称催化反应中的应用.

关链词 膦-亚磷酰胺酯配体; 不对称氢化; 不对称氢甲酰化; 不对称烯丙基烷基化; 不对称氢膦酰化; 不对称 [3+2]环加成; 不对称 1,4-加成和 1,4-还原
\end{abstract}

\section{Progress on Unsymmetrical Hybrid Chiral Phosphine- phosphoramidite Ligands and Their Application in Asymmetric Catalytic Reactions}

\author{
Hou, Chuanjin ${ }^{*, a, b} \quad$ Liu, Xiaoning ${ }^{a} \quad$ Xia, Ying $^{a} \quad$ Hu, Xiangping ${ }^{*, b}$ \\ $\left({ }^{a}\right.$ School of Light Industry and Chemical Engineering, Dalian Polytechnic University, Dalian 116034) \\ ( ${ }^{b}$ Dalian Institute of Chemical Physics, Chinese Academy of Sciences, Dalian 116023)
}

\begin{abstract}
Unsymmetrical hybrid chiral phosphine-phosphoramidite ligands have the advantages of easy accessibility, modularity and stability toward air and moisture, which make them highly appealing for asymmetric catalysis. These ligands have been found widespread utility in asymmetric catalysis, such as hydrogenation, hydroformylation, allylic alkylation, hydrophosphorylation, [3+2] cycloaddition, 1,4-addition and 1,4-reduction. The types, synthesis and applications of chiral phosphine-phosphoramidite ligands are reviewed.

Keywords phosphine-phosphoramidite ligands; asymmetric hydrogenation; asymmetric hydroformylation; asymmetric allylic alkylation; asymmetric hydrophosphorylation; asymmetric $[3+2]$ cycloaddition; asymmetric 1,4-addition and 1,4-reduction.
\end{abstract}

在过去的几十年里, 不对称催化得到了蓬勃发展, 无论是在基础研究方面, 还是在工业应用方面都取得了 显著的进步 ${ }^{[1,2]}$. 在基础研究方面, 数以千计的手性配体 被设计合成出来; 高催化活性、高光学选择性的催化剂 体系不断涌现; 新的不对称催化反应不断地被发掘; 新 的不对称催化概念相继被发展. 在工业应用方面, 氢 化、环氧化、环丙烷化等不对称催化反应在手性医药、 手性农药等多个产业中得到了成功应用.

在手性配体的设计、合成及应用领域, 手性膦配体 占据着十分重要的地位 ${ }^{[3]}$. 回顾手性膦配体的发展历程,
我们不难看出，各种不同结构的手性膦配体被设计并合 成出来, 各种配体设计的理念相继得到了发展 ${ }^{[4 \sim 6]} .20$ 世纪 70年代, DIOP [(4S,5S)-2,3-O-异丙烯基-2,3-二羟基$1,4-$ 双 (二苯基膦)丁烷 $]^{[7]}$ 和 DIPAMP $\{(S, S)$-双 $[(2$-甲氧基 苯基)苯基膦 $]$ 乙烷 $\}^{[8]}$ 的成功, 使得 $C_{2}$ 对称性的配体设 计理念深入人心, 人们发展了许多具有 $C_{2}$ 对称性的手 性双膦配体; 20 世纪 80 年代, BINAP [(R)-2,2'-双(二苯 基膦)-1,1'-联萘 $]^{[9]}$ 的兴起, 逐步推动了轴手性配体的发 展 $^{[10 \sim 15]} ; 20$ 世纪 90 年代, 随着 Duphos $(1,2$-双 [(2S,5S)2,5 -二甲基磷]苯)和 $\mathrm{BPE}\{1,2$-双 $[(2 R, 5 R)-2,5$-二甲基膦 $]$

\footnotetext{
*E-mail: houcj@dlpu.edu.cn, xiangping@dicp.ac.cn

Received May 30, 2012; revised July 17, 2012; published online July 18, 2012.

Project supported by the Planned Science and Technology Project of Dalian City (No. 2011J21DW010), and the Scientific Research Project of Department of Education of Liaoning Province (No. L2010048).

大连市科技局科技计划项目(No. 2011J21DW010)和辽宁省教育厅科学技术研究(No. L2010048)资助项目.
} 
乙烷 $]^{[16 ~ 18]}$ 配体的成功应用, 环状膦配体的设计理念得 到了大家的认可, 一些环状的磷手性中心配体被相继报 道 ${ }^{[19 ~ 23]}$. 近来的研究表明, 非对称性杂化的手性膦配体 在许多不对称催化反应中表现出与 $C_{2}$ 对称的手性双膦 配体相当甚至更为优异的活性和光学选择性. 这些非对 称杂化的手性膦配体在不对称催化反应中的成功应用, 极大的拓展了手性膦配体的设计理念, 丰富了手性膦配 体的种类. 本文对非对称杂化的手性膦-亚磷酰胺酯配 体 ${ }^{[24 ~ 27]}$ 的种类、合成及其在不对称催化反应中的应用进 展进行综述.

\section{1 膦-亚磷酰胺酯配体的种类与合成}

2000 年, Leitner 等 ${ }^{[28]}$ 报道了第一例非对称杂化的手 性膦-亚磷酰胺酯配体 QUINAPHOS (1a) (Scheme 1). 此后, 人们对其结构进行了各种改造和优化, 合成了许 多手性膦-亚磷酰胺酯配体. 该类型配体的手性主要来 源于成两个部分: 二醇部分和胺部分. 其中二醇部分多 为 $\operatorname{BINOL}$ (联萗酚), $\mathrm{H}_{8}$-BINOL, 二酚等, 胺部分包含与 金属配体的两个膦原子, 因此其结构变化比较多. 根据 配体胺部分中 $\mathrm{N}$ 原子的来源, 我们可以把膦-亚磷酰胺 酯配体分成四类: 第一类配体 (主要是源于喹啉和吲哚 等杂环骨架的配体如 QUINAPHOS, INDOLPhos 等), 第 二类配体(主要是源于二茂铁骨架的配体如 PPFAPhos 等), 第三类配体 (主要是源于苯乙胺类骨架的配体如 PEAPhos, THNAPhos 等)和第四类(其他)配体.

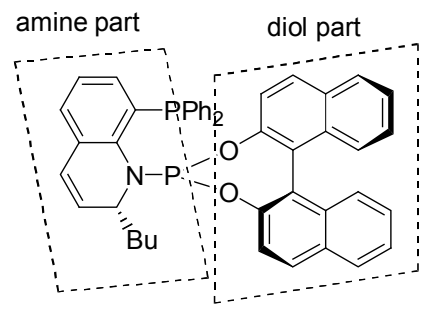

QUINAPHOS (1a)

\section{Scheme 1}

\section{1 第一类: 源于杂环骨架的膦-亚磷酰胺酯配体}

Leitner 等 ${ }^{[28]}$ 以 8-二芳基膦喹啉 2 为原料, 用有机锂 试剂锂化后得到相应的 1,2-二氢喹啉盐 $\mathbf{3}$, 再与亚磷酰 氯衍生物反应, 制备得到 QUINAPHOS 类配体 $\mathbf{1}$ (Scheme 2).

QUINAPHOS 类配体 1 的两个磷原子与中心金属配 位时可形成一个六元环的鳌合物. 而鳌合环的大小可能 会对催化反应产生很大的影响，因此 Reek 等 ${ }^{[29]}$ 在 QUINAPHOS 的基础上，合成了可以与中心金属形成五 元鳌合环的膦-亚磷酰胺酯配体 INDOLPhos (4)和 INDOLPhosphole (5) (Scheme 3), 而且该配体的金属螯

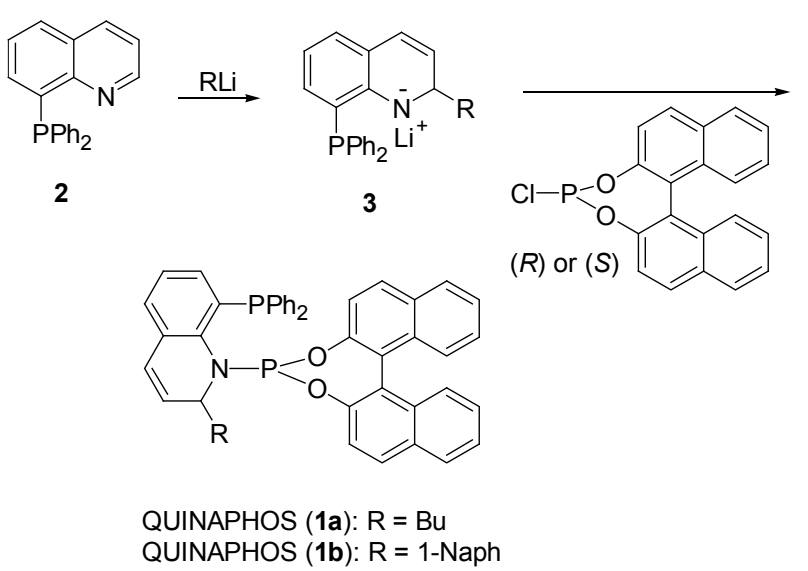

Scheme 2

合物骨架 $\mathrm{C}$ 原子均为 $\mathrm{sp}^{2}$ 杂化, 所以其刚性很强, 可以 在反应中形成好的手性环境, 有利于反应的立体选择 性.

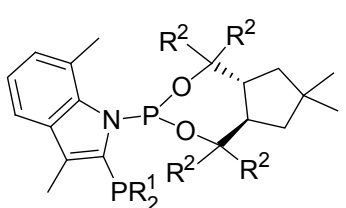

4a: $\mathrm{R}^{1}=i-\operatorname{Pr}, \mathrm{R}^{2}=\mathrm{Me}$

4c: $R^{1}=P h, R^{2}=P h$ 4b: $R^{1}=P h, R^{2}=M e$

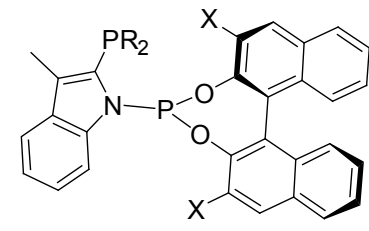

4d: $\mathrm{R}=\mathrm{Ph}, \mathrm{X}=\mathrm{H}$

4e: $\mathrm{R}=i-\mathrm{Pr}, \mathrm{X}=\mathrm{H}$

4f: $\mathrm{R}=\mathrm{Ph}, \mathrm{X}=\mathrm{SiMe}_{3}$

4g: $\mathrm{R}=i-\mathrm{Pr}, \mathrm{X}=\mathrm{Me}$

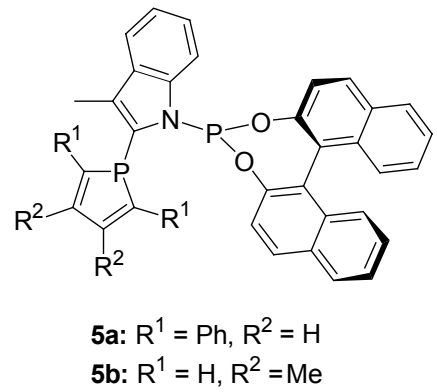

\section{Scheme 3}

\section{2 第二类: 源于二茂铁骨架的膦-亚磷酰胺酯配体}

2004 年, 我们 ${ }^{[30]}$ 和 Chan 等 ${ }^{[31]}$ 分别独立报道了含二 茂铁骨架的膦-亚磷酰胺酯配体 PPFAPhos (6)的合成 (Scheme 4). 该配体以二茂铁衍生的Ugi-胺 7 为原料, 经 邻位锂化后再引入二苯基膦得到化合物 $\mathbf{8 , 8}$ 先后与乙酸 酐和胺反应得到化合物 $\mathbf{9}$, 后者与亚磷酰氯衍生物反应, 可制得 PPFAPhos (6).

\section{3 第三类: 源于苯乙胺骨架的膦-亚磷酰胺酯配体}

由于 PPFAPhos (6)中二茂铁骨架的刚性过大, 有时 可能会对反应不利，因此，我们 ${ }^{[32]}$ 从商业易得的 $(S)$-苯 乙胺 10 出发, 经邻位锂化引入二苯基膦得中间体 11, 11 

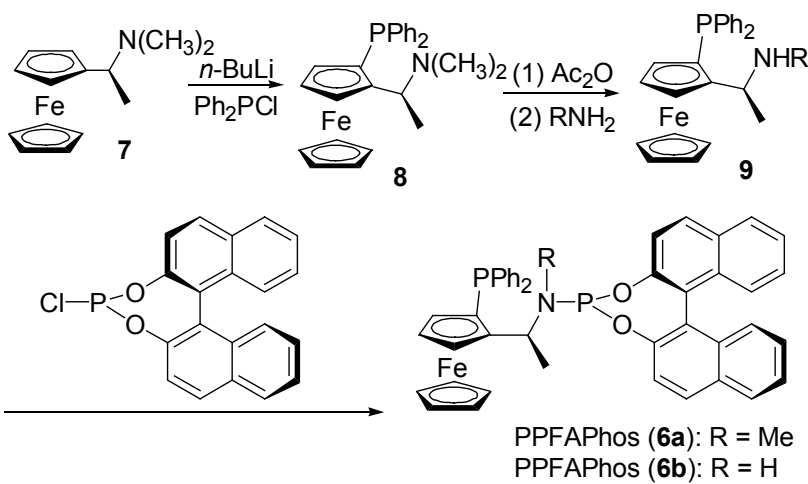

Scheme 4

与亚磷酰氯衍生物反应, 合成了 PEAPhos (12) (Scheme 5). 该配体以柔性的苯乙胺替代了二茂铁骨架, 在一些 反应中取得了非常好的结果.

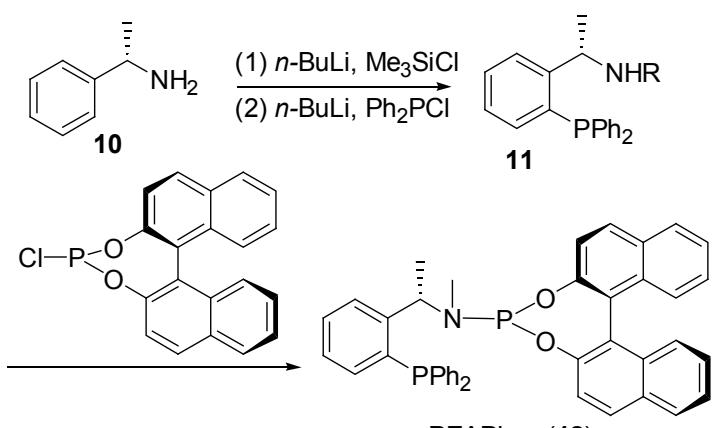

PEAPhos (12)

Scheme 5

\section{4 第四类: 其他膦-亚磷酰胺酯配体}

2006 年, Zhang 等 ${ }^{[33]}$ 从 $(S)$-BINOL (13)出发, 先在其 3,3'-位引入两个二苯基膦得化合物 14, 进而合成了一类 特殊的三齿膦-亚磷酰胺酯配体 15 (Scheme 6). 该配体 虽为三齿配体，但 $\mathrm{X}$ 单晶衍射证实，其与金属形成的螯 合物中仅有两个磷原子参与配位, 因而存在一个自由的 没有参与配位的磷原子, 为催化反应提供了多样化的手 性环境.

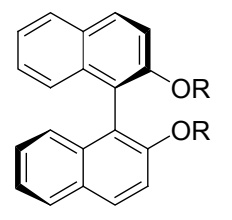

13

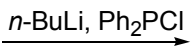

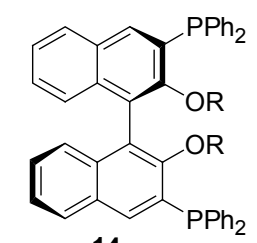

14

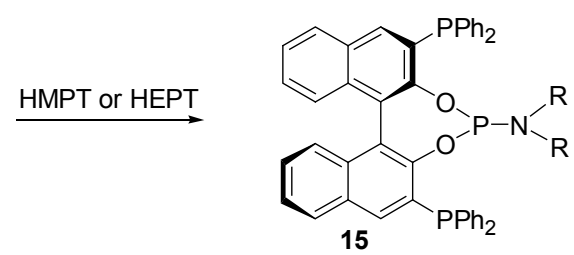

Scheme 6
2012 年, Bakos 等 ${ }^{[34]}$ 利用环状硫酸酯 $\mathbf{1 6}$ 与异丙基胺 的开环反应得到氨基磺酸 17 , 然后与二苯基膦锂反应 得到氨烷基膦 18 , 化合物 18 与亚磷酰氯衍生物反应, 得到了一例含有两个碳手性中心和一个轴手性中心的 膦-亚磷酰胺酯配体 19 (Scheme 7).

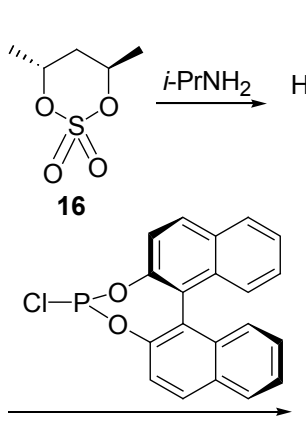

Scheme 7

\section{2 膦-亚磷酰胺酯配体在不对称催化反应中的 应用}

\section{1 膦-亚磷酰胺酯配体在不对称氢化反应中的应用}

不对称催化氢化反应，因其原子经济性高，一直备 受人们关注，是制备光学纯化合物的最有效最方便的方 法之一. 本文将着重介绍膦一亚磷酰胺酯配体在 $\alpha, \beta$-不 饱和羧酸酯、烯酰胺、脱氢氨基酸、衣康酸酯、烯醇膦 酸酯、亚胺、芳香酮等化合物的不对称氢化反应中的应 用.

我们将 $\left(R_{\mathrm{c}}, R_{\mathrm{a}}\right)$-THNAPhos (22)用于 $\alpha, \beta$-不饱和羧酸 酯 Roche 酯 20 的氢化反应 ${ }^{[35]}$, 结果表明酯基对反应的 对映选择性影响很大, 增大酯基的位阻, 产物的 $e e$ 值降 低(Eq. 1).

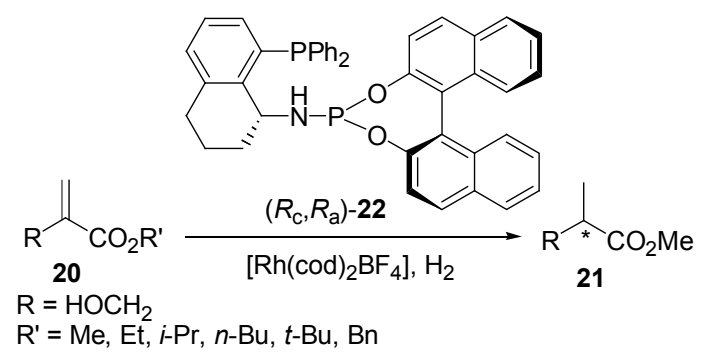

2000 年, Leitner 等 ${ }^{[28]}$ 将 QUINAPHOS 1a 用 Rh-催化 烯酰胺 23 和衣康酸二甲酯 $\mathbf{2 5}$ 的不对称催化氢化反应, 得到了 $97.8 \%$ 和 $98.8 \%$ 的对映选择性, 其中 $\left(R_{\mathrm{c}}, R_{\mathrm{a}}\right)$ 为匹 配构型(Eq. 2). 当他们采用含有柔性骨架 $1,2,3,4$-四氢喹 啉衍生的配体 ${ }^{[36]}$ 时, 却发现 $\left(S_{\mathrm{c}}, R_{\mathrm{a}}\right)$ 为匹配构型(Eq. 3), $\left(S_{\mathrm{c}}, R_{\mathrm{a}}\right)-\mathbf{1 b} / \mathrm{Rh}$ 催化 $\mathbf{2 3}$ 和 $\mathbf{2 5}$ 的不对称氢化反应, 可得到 $>99 \%$ ee 值的产物, 该反应的 TOF 高达 $20000 \mathrm{~h}^{-1}$. 


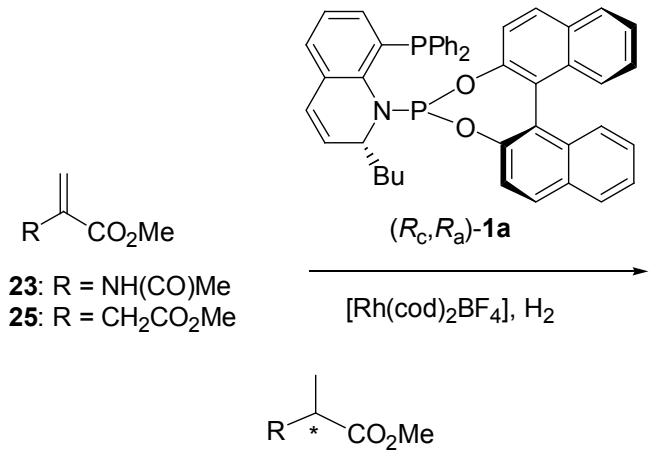

(2)

(S)-24 97.8\% ee (R)-26 98.8\% ee
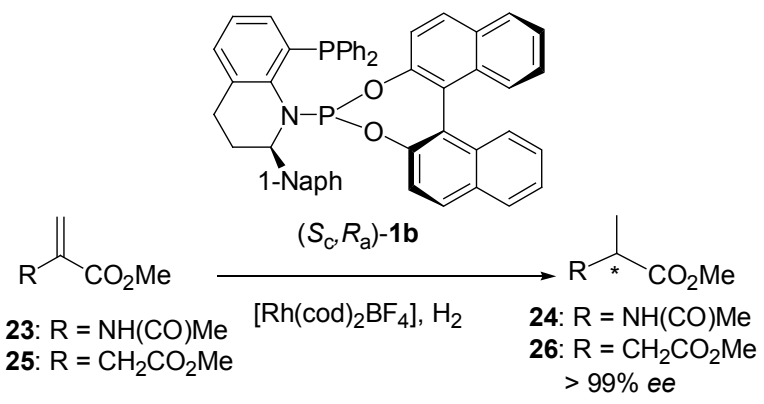

2004 年, 我们 ${ }^{[30]}$ 将 $\left(S_{\mathrm{c}}, R_{\mathrm{p}}, S_{\mathrm{a}}\right)$-PPFA (6a) 分别应用烯 酰胺 23、衣康酸二甲酯 25 及 $\alpha$-脱氢氨基酸酯 $\mathbf{2 7}$ 的氢 化反应, 最高均可得到 $>99 \%$ 的对映选择性(Scheme 8 ). 尤其对于衣康酸二甲酯 $\mathbf{2 5}$ 的氢化, 在 $S / C=10000$ 的条 件下，依然取得了 99.1 的 $e e$ 值.<smiles>C=C(NC(C)=O)c1ccccc1</smiles><smiles></smiles><smiles>CC(=O)NC(C)c1ccccc1</smiles>

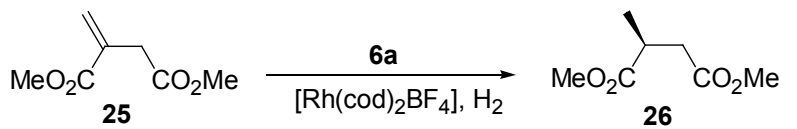<smiles>CCCNC(=Cc1ccccc1)C(C)=O</smiles>

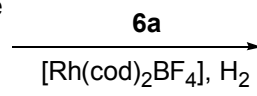<smiles>CC(=O)N[C@@H](Cc1ccccc1)C(C)=O</smiles><smiles>CC(C1CCCC1)N(C)P(Oc1ccc2ccccc2c1-c1c(OP(C)c2ccccc2)ccc2ccccc12)C1CCCC1</smiles>

$\left(S_{\mathrm{c}}, R_{\mathrm{p}}, S_{\mathrm{a}}\right)-\operatorname{PPFA}(\mathbf{6 a})$

Scheme 8

对于 $\beta$-脱氢氨基酸酯 29 的氢化反应, 我们发现配 体中 $\mathrm{N}$ 原子的上取代基对反应的立体选择性至关重 要 ${ }^{[37]}$. 采用 $\mathrm{NCH}_{3}$ 取代的配体 $\mathbf{6 a}$ 时, 产物的 $e e$ 值为
$65 \%$; 而采用 $\mathrm{NH}$ 取代的配体 $\mathbf{6 b}$ 时，其 $e e$ 值高达 $98 \%$. 该法对于 $\beta$-烷基和 $\beta$-芳基取代的 $\beta$-脱氢氨基酸酯均能 很好的反应, $e e$ 值为 $96 \% \sim 99 \%$ (Eq. 4).

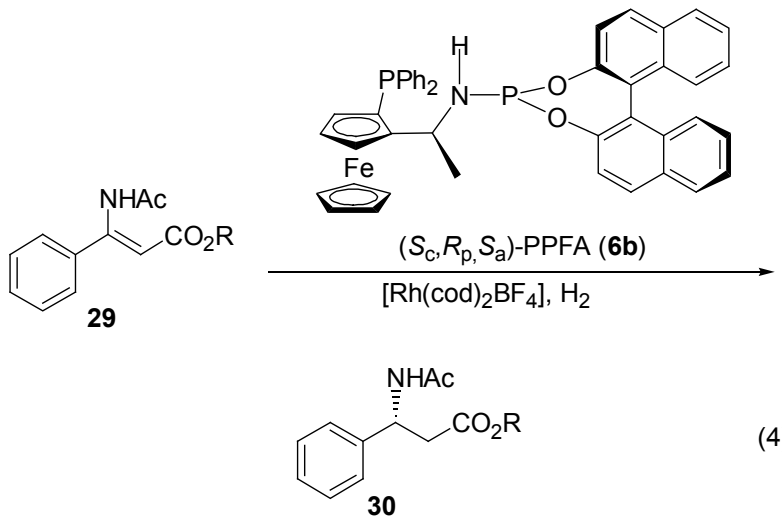

考虑二茂铁骨架的刚性，我们在 PPFAPhos 6 的基 础上, 合成了含有柔性苯乙胺骨架的配体 PEAPhos

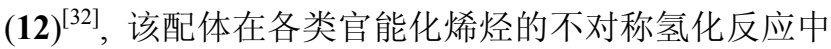
均取得了优异的结果. 尤其是对于 $\beta$-脱氢氨基酸酯 29 的氢化反应，我们发现配体 BINOL 上 3,3'-位取代基的 位阻效应，对反应影响很大 ${ }^{[38]}$, 增大取代基的位阻，有 利于提高反应的对映选择性(Eq. 5).

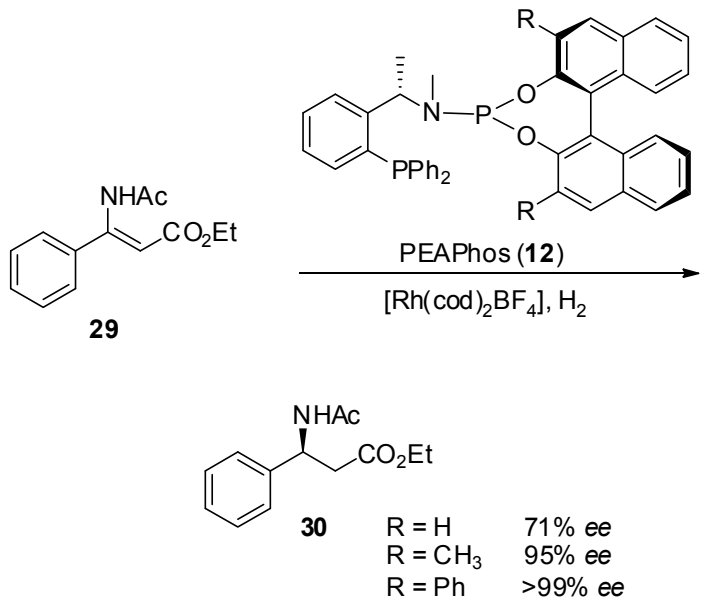

2006 年, Kostas 等 ${ }^{[39]}$ 以无手性中心的苯胺骨架代替 手性的苯乙胺骨架, 合成了一个膦一亚磷酰胺酯配体 Me-AnilaPhos (31). 将其用于 $\alpha$-脱氢氨基酸酯 27 的氢 化, 反应在 $1 \mathrm{~mol} \% \mathrm{Rh} / \mathbf{3 1}$ 催化剂下, 常温常压反应 10 $\min$ 即可完全转化, 产物的 $e e$ 值为 $97.9 \%$ (Eq. 6).

Leitner 等 ${ }^{[40]}$ 对 Me-AnilaPhos (31)配体结构进行了 改造，合成了配体 32. 该配体在 $\alpha$-脱氢氨基酸酯 $\mathbf{2 7}$ 的 氢化反应中可得到 $>99 \%$ 的对映选择性. 此外，作者将 其应用于 Ir-催化喹啉 $\mathbf{3 3}$ 的不对称催化氢化反应中，同 样取得了不错的结果(Eq. 7). 


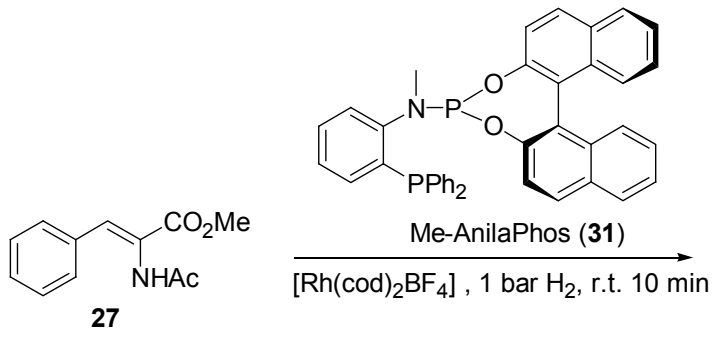

(6)

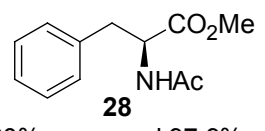

$100 \%$ conv. and $97.9 \%$ ee
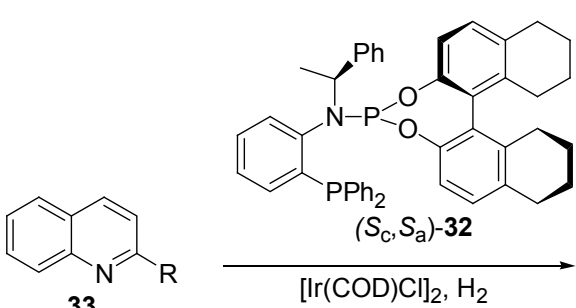

$\left(S_{\mathrm{c}}, S_{\mathrm{a}}\right)-32$

$\left[\operatorname{lr}(\mathrm{COD}) \mathrm{Cl}_{2}, \mathrm{H}_{2}\right.$<smiles>[R]C1CCc2ccccc2N1</smiles>

34

$95 \% \sim 97 \%$ ee

传统的氢化反应大多使用二氯甲烷等有毒的溶剂, 而使用环境友好的溶剂 ${ }^{[41]}$ (如碳酸酯、乙醇、水等)或不 使用溶剂的有机反应，是绿色化学发展的方向. 近来， Bakos 等 ${ }^{[34]}$ 使用环境友好的有机碳酸酯作为溶剂, 考察 了膦-亚磷酰胺酯配体 19 在脱氢氨基酸酯 27 的氢化反 应中的应用, 反应的对映选择性高达 99\% (Eq. 8). 重要 的是, 对于化合物 $27 \mathrm{a}$ 的氢化, 仅通过简单的过滤、洗 涤纯化, 便可得到 $71 \%$ 收率和 $99.6 \%$ 光学纯度的产物 28a, 化合物 28a 可用于合成治疗帕金森综合症的药物 $L$-dopa.

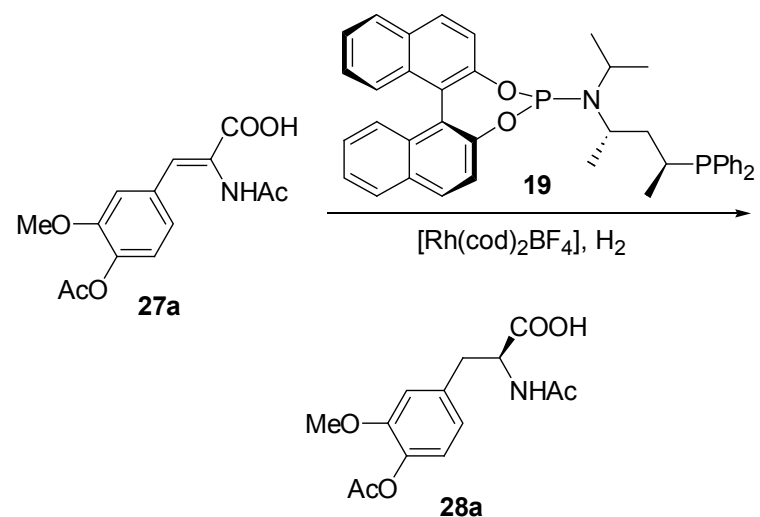

2006 年, Zhang 等 ${ }^{[42]}$ 将三齿膦-亚磷酰胺酯配体 $\mathbf{1 5}$ 用于官能化烯烃的氢化反应，值得注意的是该配体在一 些挑战性的三取代烯酰胺 35 和邻取代芳基烯酰胺 37 的
氢化反应中，取得了前所未有的好结果(Scheme 9).

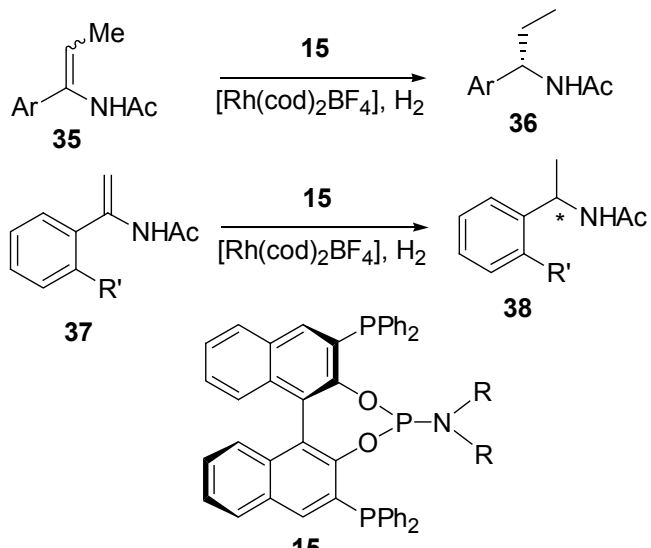

Scheme 9

Reek 等 ${ }^{[43,44]}$ 将 Taddol 衍生的 IndolPhos 配体 $4 \mathbf{a}$ 和 4b 用于烯醇膦酸酯 39a 的不对称氢化反应时, 发现使用 4a 作配体时可得到完全转化和中等的 $e e$ 值, 而使用作 4b 配体时, 转化率和对映选择性均非常差(Eq. 9).

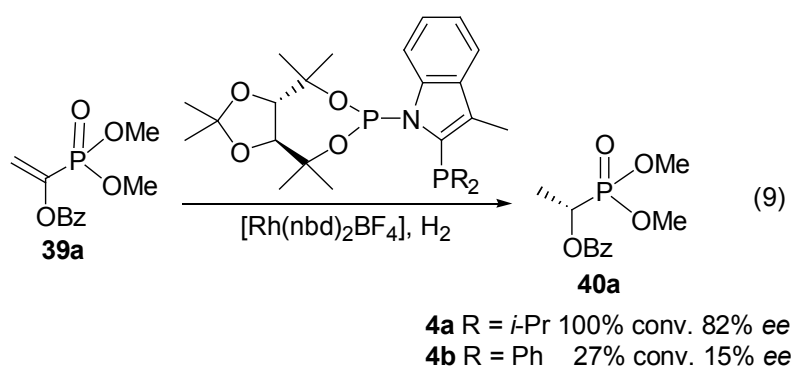

考虑到 PPFAPhos 配体中二茂铁骨架刚性过强, 而 PEAPhos 中苯乙胺骨架过柔，我们希望能寻找到一个刚 柔性适中的配体骨架. 2007 年, 我们 ${ }^{[45,46]}$ 以四氢荟胺为 原料合成了配体 THNAPhos (22), 该配体在 $\beta$-芳基、 $\beta$ 烷基、 $\beta$-烷氧基烯醇膦酸酯 39 的氢化反应中，均可得到 $>99 \%$ 的 $e e$ 值(Eq. 10), 这是该类底物氢化反应中最高 效、适用范围最广的催化体系，对手性膦酸酯的合成和 工业应用具有重要意义.

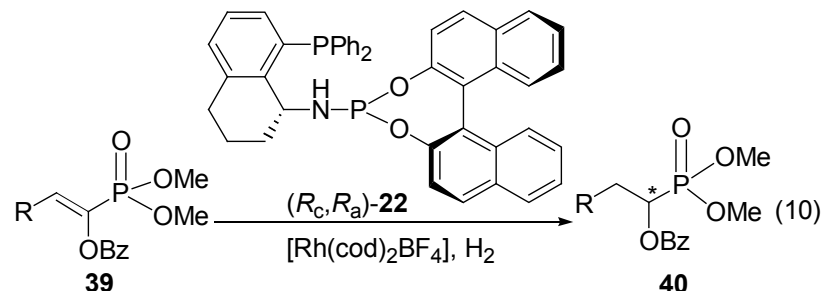

2008 年，我们 ${ }^{[47]}$ 又采用价格便宜的 1-荟胺代替昂 贵的四氢菜胺，合成了配体 $(S)-H Y-P h o s ~(41)$, 该配体虽 然比 THNAPhos (22) 少了 1 个碳手性中心, 但在烯醇膦 酸酯 39 的氢化反应中，依然可以得到最高 $99 \%$ 的对映 
选择性(Eq. 11).

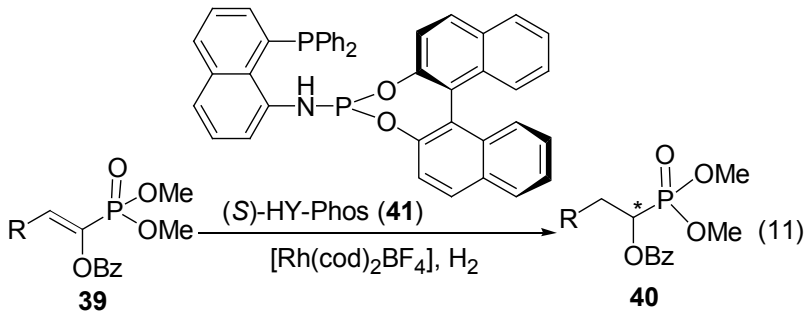

此外, Leitner 等 ${ }^{[48]}$ 将 QUINAPHOS (1a) 用于 Ru-催 化芳香酮 42 的不对称催化氢化反应(Eq. 12), 反应以为 QUINAPHOS (1a)配体、 $\left[\mathrm{RuCl}_{2}\left(\mathrm{C}_{6} \mathrm{H}_{6}\right)\right]_{2}$ 为金属前体、二 胺 44 或 45 为助催化剂. 结果发现配体的构型对反应的 对映选择性均有很大影响, $\left(S_{\mathrm{c}}, R_{\mathrm{a}}\right)$ 为匹配的构型. 另外, 助催化剂二胺同样影响反应的对映选择性, 使用乙二胺 44 为助催化剂时, 产物的 $e e$ 值最高为 $82 \%$; 而使用手性 的 $(S, S)-1,2$-二苯基乙二胺 (45) 为助催化剂时, 产物的 $e e$ 值可达 $94 \%$.
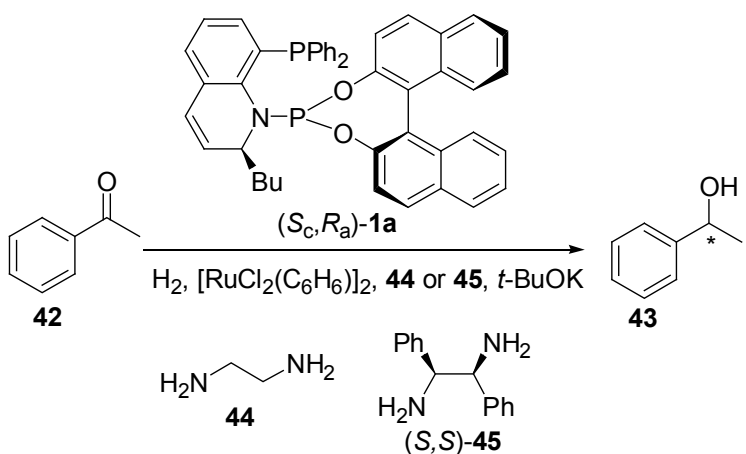

\section{2 膦-亚磷酰胺酯配体在不对称氢甲酰化反应中的} 应用

不对称氢甲酰化反应是通过烯烃与氢气和一氧化碳 混合气体反应，从而在烯烃分子中引入甲酰基，同时又 形成新的手性中心. 这是不对称催化中研究较多, 也是 有机化学非常感兴趣的反应之一.

Leitner 等 ${ }^{[28]}$ 将 QUINAPHOS (1a)用于 Rh-催化苯乙 烯 46 的不对称氢甲酰化反应(Eq. 13). 结果表明产物的 $e e$ 值取决于杂环骨架中 2 号位 C 原子的绝对构型：使用 配体 $\left(S_{\mathrm{c}}, R_{\mathrm{a}}\right)-1 \mathrm{a}$ 时, 可得到 74\% ee 的 $(S)$-型产物; 而使用 配体 $\left(R_{\mathrm{c}}, R_{\mathrm{a}}\right)-1 \mathrm{a}$ 时, 得到消旋的产物.

Reek 等 ${ }^{[49]}$ 将 IndolPhos 配体 $\mathbf{4 d} \sim \mathbf{4 g}$ 用于苯乙烯 46 的不对称氢甲酰化反应, 得到了较好的区域选择性和立 体选择性(Eq. 14). 其中支链产物 $\mathbf{4 7}$ 和直链产物 $\mathbf{4 8}$ 的比 值 $(b / 1)$ 最高为 $17: 1$, 产物 $\mathbf{4 7}$ 的 $e e$ 值最高为 $72 \%$. 此后, 他们又将 Taddol 衍生的 IndolPhos 配体 $\mathbf{4 a}$ 和 $\mathbf{4 b}$ 用于不 对称氢甲酰化反应 ${ }^{[50]}$, 对于苯乙烯、烯醇酯和烯丙基氰 等底物分别得到了最高为 $72 \%, 74 \%$ 和 $63 \%$ 的对映选择
性.

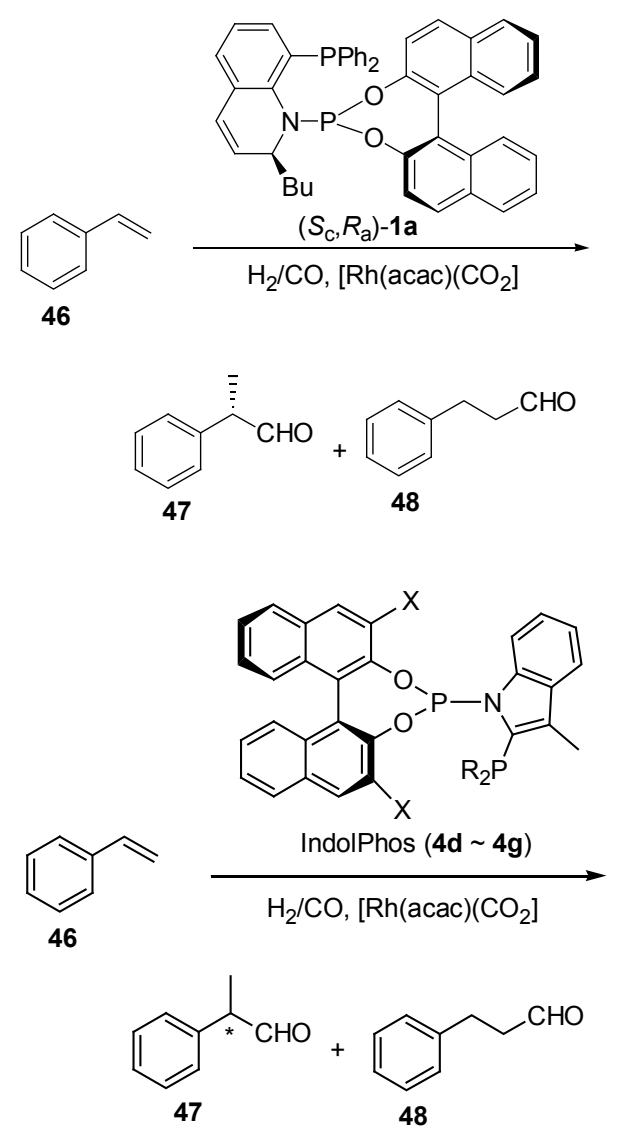

近来, Reek 等 ${ }^{[51]}$ 将 INDOLPhos (4c)用于挑战性的底 物 2,3-二氢呋喃 49 的不对称氢甲酰化反应(Eq. 15), 2-取 代产物 50 和 3-取代产物 51 的比例为 $68: 32$, 其中产物 50 的 $e e$ 值为 $62 \%$. 该方法通过 49 的氢甲酰化反应，首 次以较高的区域选择性和立体选择性的合成了化合物 50.

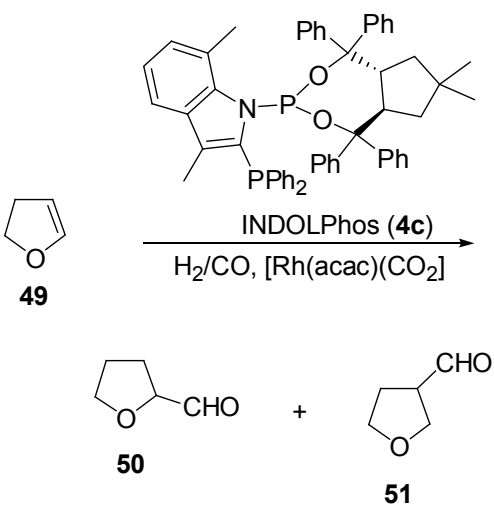

2006 年, Zhang 等 ${ }^{[2]}$ 从手性 NOBIN 出发, 报道了 Yanphos 配体 $(R, S)$-52 的合成, 并将其应用于苯乙烯衍 生物 46 的不对称氢甲酰化反应(Eq. 16). 反应中观察到 很强的溶剂效应，非极性的苯和二氯甲烷效果最佳，反 应的立体选择性最高达 $99 \%$ ee . 值得注意的是，延长反 
应时间并没有发现 Binaphos 配体中的消旋化现象 ${ }^{[53]}$. 此后, 作者又详细研究了该类型配体在苯乙烯、烯醇酯 和烯丙基氰等底物的不对称氢甲酰化反应, 结果发现配 体的位阻效应对反应影响很大 ${ }^{[54]}$.
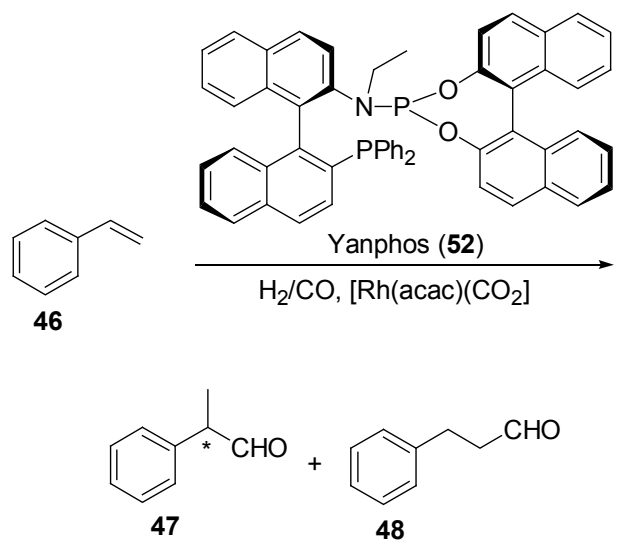

2010 年, Zhang 等 ${ }^{[55]}$ 首次将 $N$-烯丙基酰胺(磺酰 胺)53 用于 Yanphos 52/Rh 催化的不对称氢甲酰化反应 (Eq. 17), 得到了一系列手性 $\beta^{2}$-氨基醛, 其中的醛基通 过进一步反应可转化为羧基和羟基, 提供为了一种制备 手性 $\beta^{2}$-氨基醛、酸、醇的好方法.

$$
\begin{aligned}
& \mathrm{R}^{1} \backslash \mathrm{N} \\
& \mathrm{R}^{2} \\
& 53
\end{aligned}
$$

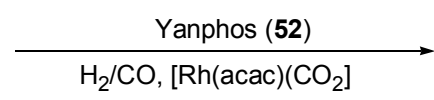

$\mathrm{R}^{1}=\mathrm{Boc}, \mathrm{Bz}, \mathrm{Ts}$

$\mathrm{R}^{2}=\mathrm{H}, \mathrm{Me}, \mathrm{Boc}$

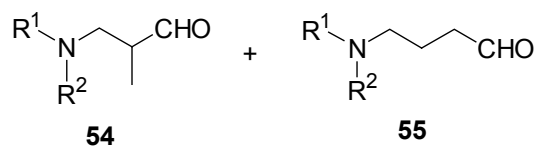

\section{3 膦一亚磷酰胺酯配体在不对称烯丙基烷基化反应 中的应用}

2009 年, Reek 等 ${ }^{[56,57]}$ 将 IndolPhos 配体 $\mathbf{4 d} \sim \mathbf{4 g}$ 与 $\left[\mathrm{Pd}_{2}\left(\eta^{3}-\mathrm{C}_{3} \mathrm{H}_{5}\right)_{2} \mathrm{Cl}_{2}\right]$ 原位生成的催化剂用于单取代和二取 代烯丙醇乙酸酯 56 的烯丙基烷基化反应(Scheme 10). 对于 1,3-二苯基-2-丙烯醇乙酸酯(56a), 最高可得到 $90 \%$ 的 $e e$ 值. 对于单取代的肉桂醇乙酸酯 $\mathbf{5 6 b}$, 虽然其区域 选择性较低 $(b / l=14: 86)$, 但可得到较好的对映选择性 $(81 \%$ ee).

\section{4 膦-亚磷酰胺酯配体在不对称氢膦酰化反应中的 应用}

2005 年, Leitner 等 ${ }^{[58]}$ 报道了过渡金属催化的芳香烯 烃 60 与频哪醇 $H$-膦酸酯 61 的氢膦酰化反应(Eq. 18). 采 用 Rh 催化剂(如 Wilkinson 催化剂)时, 仅得到反马氏规
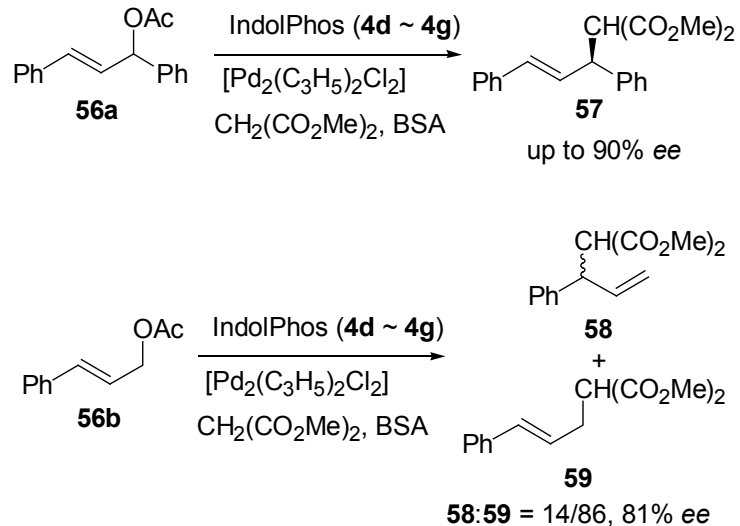

Scheme 10

则的产物 62; 采用 $\mathrm{Pd}$ 催化剂时可以高选择性的得到马 氏规则的产物 63. 使用手性配体 $\left(S_{\mathrm{c}}, R_{\mathrm{a}}\right)$-QUINAPHOS $1 \mathrm{a}$ 时, 马氏与反马氏产物比例为 $87: 13$, 产物 63 的 $e e$ 值为 $10 \%$. 虽然产物 $e e$ 值很低, 但这是首例不对称的氢 膦酰化反应.

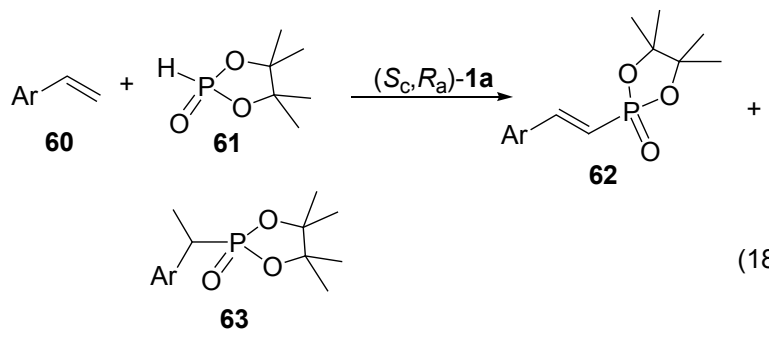

2.5 膦一亚磷酰胺酯配体在不对称 $[3+2]$-环加成反应 中的应用

2009 年, 我们 ${ }^{[59]}$ 将二茂铁衍生的膦-亚磷酰胺酯配 体 PPFAPhos (6) 用于 Ag-催化亚甲胺叶立德 64 与马来酸 二甲酯 65 的不对称[3+2]-环加成反应(Eq. 19). 结果表 明使用含有磷手性中心的配体 $\left(S_{\mathrm{c}}, R_{\mathrm{Fc}}, S_{\mathrm{a}}, R_{\mathrm{P}}\right)$-PPFA (6c) 时,可获得最佳的非对映选择性(endo/exo 最高为 99/1) 和 对映选择性(最高 $99 \%$ ee 值).

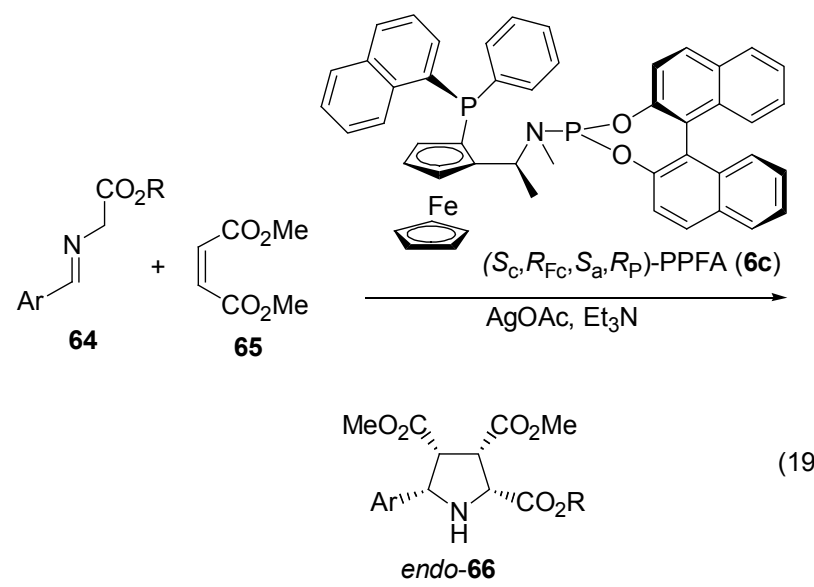




\section{6 膦-亚磷酰胺酯配体在不对称 1,4-加成和 1,4-还原 反应中的应用}

2006 年, Crévisy 等 ${ }^{\left[{ }^{[00]}\right.}$ 以 $\beta$-氨基醇为原料, 报道了 膦-亚磷酰胺酯配体 67 的合成并将其应用于 $\mathrm{Cu}$ 催化二 乙基锌与环已烯酮的 1,4-加成反应(Eq. 20). 虽然该反应 仅得到了 $53 \% \mathrm{ee}$ 值的产物, 但这却是膦-亚磷酰胺酯配 体在 1,4-加成反应中的首次应用.

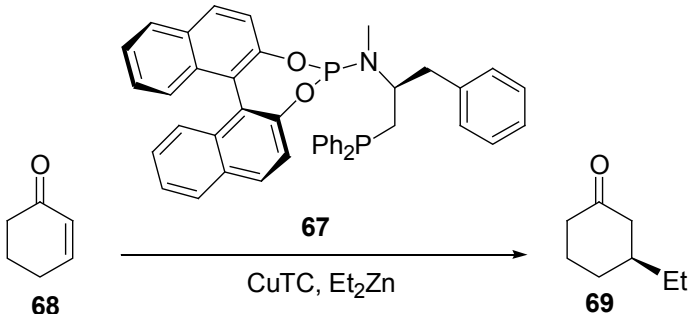

2011 年, 我们 ${ }^{[61]}$ 将二茂铁衍生的膦-亚磷酰胺酯配 体 PPFAPhos (6) 用于 $\mathrm{Cu}$ 催化 $\alpha, \beta$-不饱和酯 70 的 1,4-还 原反应(Eq. 21). 值得注意的是, 配体的构型对反应结果 有很大影响. 在不对称氢化中取得良好结果的配体 $\left(S_{\mathrm{c}}, R_{\mathrm{p}}, S_{\mathrm{a}}\right)$-PPFA (6a) 仅得到较差的对映选择性, 而在不 对称氢化中不匹配的配体 $\left(S_{\mathrm{c}}, R_{\mathrm{p}}, R_{\mathrm{a}}\right)-$-PPFA $(\mathbf{6 d})$ 取得了最 好的结果, 产物的 $e e$ 值最高可达 $99 \%$.

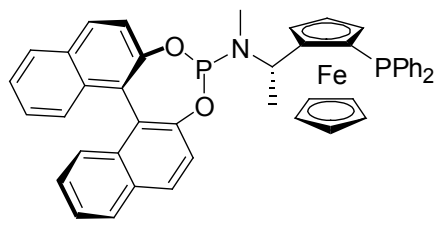<smiles>[R]C(=O)C(=O)O[K]</smiles>

$\left(S_{\mathrm{C}}, R_{\mathrm{p}}, R_{\mathrm{a}}\right)$-PPFAPhos $(\mathbf{6 d})$

$\mathrm{CuF}\left(\mathrm{PPh}_{3}\right)_{3} \cdot 2 \mathrm{MeOH}, \mathrm{PMHS}, t-\mathrm{BuOH}$<smiles>[R]OC(=O)CC([R])[Al]</smiles>

\section{3 结束语}

由于手性膦-亚磷酰胺酯配体中的两个磷原子是非 对称杂化的, 可以在反应中提供非常丰富的手性环境, 有利于提高不对称催化反应的立体选择性, 因而手性 膦一亚磷酰胺酯配体已经在不对称催化氢化、不对称氢 甲酰化、不对称烯丙基烷基化、不对称氢膦酰化、不对 称[3+2]-环加成、不对称 1,4-加成和 1,4 还原等反应中 有着广泛的应用. 另外由于配体中两个非等价的磷配位 原子是在配体合成中分步引入的, 这为后续配体结构的 优化、调控提供了更为便利的条件. 通过分别调节两个 配位原子上的电子和空间性质, 可以合成具有独特结构
特征、满足特定催化反应要求的高效手性膦-亚磷酰胺 酯配体．尽管如此，我们可以看到手性膦-亚磷酰胺酯 配体依然存在着普适性差的缺点, 大多数配体仅在不对 称氢化和不对称氢甲酰化反应中有着很好的应用, 而在 其他类型的反应中应用较少. 此外，个别手性膦-亚磷 酰胺酯配体存在着合成困难、价格昂贵等问题，使得其 应用受到限制. 因而设计并合成一些制备简便、价格低 廉、结构稳定、底物普适性好的非对称杂化的手性 膦 一亚磷酰胺酯配体，仍然是目前不对称催化研究领域的 核心内容之一. 近来, 我们 ${ }^{[62]}$ 首次将 PEAPhos 类膦-亚 磷酰胺酯配体应用于 Ir-催化高位阻亚胺的不对称氢化 反应，取得了非常好的结果. 在此基础上，我们尝试将 其应用于手性农药 $(S)$-异丙甲草胺的制备，结果发现该 类配体对关键中间体亚胺的氢化具有很高的活性，在 $S / C=100000$ 时，显示出了与 Xyliphos ${ }^{[63,64]}$ 类似的活性 和更高的立体选择性. 因此，我们相信，未来膦一亚磷酰 胺酯配体在基础方面及工业方面都将有着更加广阔的 应用。

\section{References}

[1] Jacobsen, E. N.; Pfaltz, A.; Yamamoto, H. Comprehensive Asymmetric Catalysis, Springer, Berlin, 1999.

[2] Ojima, I. Catalytic Asymmetric Synthesis, Wiley-VCH, Weinheim, 2000.

[3] Thommen, M.; Hlaser, H.-U. In Phosphorus Ligands in Asymmetric Catalysis, Ed.: Brner A., Wiley-VCH, Weinheim, 2008

[4] Tang, W.; Zhang, X. Chem. Rev. 2003, 103, 3029.

[5] Blaser, H.-U.; Malan, C.; Pugin, B.; Spindler, F.; Steiner, H.; Studer, M. Adv. Synth. Catal. 2003, 345, 103.

[6] Zhang, W.; Chi, Y.; Zhang, X. Acc. Chem. Res. 2007, 40, 1278.

[7] Dang, T. P.; Kagan, H. B. J. Chem. Soc., Chem. Commun. 1971, 481.

[8] Vineyard, B. D.; Knowles, W. S.; Sabacky, M. J.; Bachman, G. L.; Weinkauff, D. J. J. Am. Chem. Soc. 1977, 99, 5946.

[9] Miyashita, A.; Yasuda, A.; Takaya, H.; Toriumi, K.; Ito, T.; Souchi, T.; Noyori, R. J. Am. Chem. Soc. 1980, 102, 7932.

[10] Berthod, M.; Migani, G.; Woodward, G.; Lemaire, M. Chem. Rev. 2005, 105, 1801.

[11] Ding, K.; Guo, H.; Li, X.; Yuan, Y.; Wang, Y. Top. Catal. 2005, 35, 105.

[12] Ding, K.; Li, X.; Ji, B.; Guo, H.; Kitamura, M. Curr. Org. Synth. 2005, 2, 499 .

[13] Wang, C.-J.; Liang, G.; Xue, Z.-Y.; Gao, F. J. Am. Chem. Soc. 2008, 130,17250 .

[14] Tang, W.; Xu, L.; Fan, Q.-H.; Wang, J.; Fan, B.; Zhou, Z.; Lam, K.-H.; Chan, A. S. C. Angew. Chem., Int. Ed. 2009, 48, 9135.

[15] Arshad, N.; Kappe, C. O. Adv. Heterocycl. Chem. 2010, 99, 33.

[16] Burk, M. J. J. Am. Chem. Soc. 1991, 113, 8518.

[17] Burk, M. J.; Feaster, J. E.; Nugent, W. A.; Hariow, R. L. J. Am. Chem. Soc. 1993, 115, 10125.

[18] Burk, M. J.; Bienewald, F.; Harris, M.; Zanotti-Gerosa, A. Angew. Chem., Int. Ed. 1998, 37, 1931.

[19] Tang, W.; Zhang, X. Angew. Chem., Int. Ed. 2002, 41, 612.

[20] Liu, D.; Zhang, X. Eur. J. Org. Chem. 2005, 646.

[21] Shang, G.; Yang, Q.; Zhang, X. Angew. Chem., Int. Ed. 2006, 45, 
6360 .

[22] Gridnev, I. D.; Imamoto, T.; Hoge, G.; Kouchi, M.; Takahashi, H. J. Am. Chem. Soc. 2008, 130, 2560.

[23] Zhang, X.; Huang, K.; Hou, G.; Cao, B.; Zhang, X. Angew. Chem., Int. Ed. 2010, 49, 6421.

[24] Boeda, T.; Beneyton, T.; Crévisy, C. Mini-Rev. Org. Chem. 2008, 5, 96.

[25] Eberhardt, L.; Armspach, D.; Harrowfield, J.; Matt, D. Chem. Soc. Rev. 2008, 839.

[26] Hu, X.-P.; Wang, D.-S.; Yu, C.-B.; Zhou, Y.-G.; Zheng, Z. Top. Organomet. Chem. 2011, 36, 343.

[27] Wassenaar, J.; Reek, J. N. H. Org. Biomol. Chem. 2011, 9, 1704.

[28] Franciò, C.; Faraone, F.; Leitner, W. Angew. Chem., Int. Ed. 2000, $39,1428$.

[29] Wassenaar, J.; van Zutphen, S.; Mora, G.; Floch, P. L.; Siegler, M. A.; Spek, A. L.; Reek, J. N. H. Organometallics 2009, 28, 2724.

[30] Hu, X.-P.; Zheng, Z. Org. Lett. 2004, 6, 3585.

[31] Jia, X.; Li, X.; Lam, W. S.; Kok, S. H. L.; Xu, L.; Lu, G.; Yeung, C.-H.; Chan, A. S. C. Tetrahedron: Asymmetry 2004, 15, 2273.

[32] Huang, J.-D.; Hu, X.-P.; Duan, Z.-C.; Zeng, Q.-H.; Yu, S.-B.; Deng, J.; Wang, D.-Y.; Zheng, Z. Org. Lett. 2006, 8, 4367.

[33] Zhang, W.; Zhang, X. Angew. Chem., Int. Ed. 2006, 45, 5515.

[34] Balogh, S.; Farkas, G.; Madarász, J.; Szöllősy, Á.; Kovács, J.; Darvas, F.; Ürge, L.; Bakos, J. Green. Chem. 2012, 14, 1146.

[35] 35 Qiu, M.; Wang, D.-Y.; Hu, X.-P.; Huang, J.-D.; Yu, S.-B.; Deng, J.; Duan, Z.-C.; Zheng, Z. Tetrahedron: Asymmetry 2009, 20, 210.

[36] Pullmann, T.; Engendahl, B.; Zhang, Z.; Hoelscher, M.; ZanottiGerosa, A.; Dyke, A.; Francio, G.; Leitner, W. Chem. Eur. J. 2010, 16, 7517.

[37] Hu, X.-P.; Zheng, Z. Org. Lett. 2005, 7, 419.

[38] Zhou, X.-M.; Huang, J.-D.; Luo, L.-B.; Zhang, C.-L.; Hu, X.-P.; Zheng, Z. Org. Biomol. Chem. 2010, 8, 2320.

[39] Vallianatou, K. A.; Kostas, I. D.; Holz, J.; Börner, A. Tetrahedron Lett. 2006, 47, 7947.

[40] Eggenstein, M.; Thomas, A.; Theuerkauf, J.; Francio, G.; Leitner, W. Adv. Synth. Catal. 2009, 351, 725.

[41] Schäffner, B.; Schäffner, F.; Verevkin, S. P.; Börner, A. Chem. Rev. 2010, 110, 4554.

[42] Zhang, W.; Zhang, X. J. Org. Chem. 2007, 72, 1020.

[43] Wassenaar, J.; Reek, J. N. H. J. Org. Chem. 2009, 74, 8403.
[44] Wassenaar, J.; Kuil, M.; Lutz, M.; Spek, A. L.; Reek, J. N. H. Chem. Eur. J. 2010, 16, 6509.

[45] Wang, D.-Y.; Hu, X.-P.; Huang, J.-D.; Deng, J.; Yu, S.-B.; Duan, Z.-C.; Xu, X.-F.; Zheng, Z. Angew. Chem., Int. Ed. 2007, 46, 7810.

[46] Qiu, M.; Hu, X.-P.; Wang, D.-Y.; Deng, J.; Huang, J.-D.; Yu, S.-B.; Duan, Z.-C.; Zheng, Z. Adv. Synth. Catal. 2008, 350, 1413.

[47] Yu, S.-B.; Huang, J.-D.; Wang, D.-Y.; Hu, X.-P.; Deng, J.; Duan, Z.-C.; Zheng, Z. Tetrahedron: Asymmetry 2008, 19, 1862.

[48] Burk, S.; Franciò, G.; Leitner, W. Chem. Commun. 2005, 3460.

[49] Wassenaar, J.; Reek, J. N. H. Dalton Trans. 2007, 3750.

[50] Wassenaar, J.; de Bruin, B.; Reek, J. N. H. Organometallics 2010 , $29,2767$.

[51] Chikkali, S. H.; Bellini, R.; de Bruin, B.; van der Vlugt, J. I.; Reek, J. N. H. J. Am. Chem. Soc. 2012, 134, 6607.

[52] Yan, Y.; Zhang, X. J. Am. Chem. Soc. 2006, 128, 7198.

[53] Nozaki, K.; Sakai, N.; Nanno, T.; Higashijima, T.; Mano, S.; Horiuchi, T.; Takaya, H. J. Am. Chem. Soc. 1997, 119, 4413.

[54] Zhang, X.; Cao, B.; Yan, Y.; Yu, S.; Ji, B.; Zhang, X. Chem Eur. J. 2010, 16, 871.

[55] Zhang, X.; Cao, B.; Yu, S.; Zhang, X. Angew. Chem., Int. Ed. 2010, 49, 4047.

[56] Wassenaar, J.; van Zutphen, S.; Mora, G.; Floch, L. P.; Siegler, M. A.; Spek, A. L.; Reek, J. N. H. Organometallics 2009, 28, 2724.

[57] Wassenaar, J.; Jansen, E.; van Zeist, W.-J.; Bickelhaupt, F. M.; Siegler, M. A.; Spek, A. L.; Reek, J. N. H. Nat. Chem. 2010, 2, 417.

[58] Shulyupin, M. O.; Franciò, G.; Beletskaya, I. P.; Leitner, W. Adv. Synth. Catal. 2005, 347, 667.

[59] Yu, S.-B.; Hu, X.-P.; Deng, J.; Wang, D.-Y.; Duan, Z.-C.; Zheng, Z. Tetrahedron: Asymmetry 2009, 20, 621.

[60] Boeda, F.; Rix, D.; Clavier, H.; Crévisy, C.; Mauduit, M. Tetrahedron: Asymmetry 2006, 17, 2726.

[61] Hou, C.-J.; Guo, W.-L.; Hu, X.-P.; Deng, J.; Zheng, Z. Tetrahedron: Asymmetry 2011, 22, 195.

[62] Hou, C.-J.; Wang, Y.-H.; Zheng, Z.; Xu, J.; Hu, X.-P. Org. Lett. 2012, 14, 3554.

[63] Blaser, H.-U.; Buser, H.-P.; Loers, K.; Hanreich, R.; Jalett, H. P.; Jelsch, E.; Pugin, B.; Schneider, H. D.; Spindler, F.; Wagmann, A. Chimia 1999, 53, 275.

[64] Blaser, H.-U.; Malan, C.; Pugin, B.; Spinder, F.; Steiner, H.; Studer, M. Adv. Synth. Catal. 2003, 345, 103.

(Qin, X.) 